\title{
Towards Generalized Riesz Systems Theory
}

\author{
Alan Kamuda ${ }^{1} \cdot$ Sergiusz Kużel $^{1}$ \\ Received: 4 October 2019 / Accepted: 9 February 2020 / Published online: 17 February 2020 \\ (c) The Author(s) 2020
}

\begin{abstract}
Pseudo-Hermitian Hamiltonians have recently become a field of wide investigation. Originally, the generalized Riesz systems (GRS) have been introduced as an auxiliary tool in this theory. In contrast, the current paper, GRSs are analysed in terms of basis theory. The relationship between semi-regular sequences and GRSs is provided. Various characterizations of GRSs are discussed.
\end{abstract}

Keywords Generalized Riesz system · Bi-orthogonal sequence · Bessel sequence · Friedrichs extension $\cdot$ Self-adjoint operator

Mathematics Subject Classification Primary 47B25; Secondary 47N50 • 47B65

\section{Introduction}

Theory of non self-adjoint operators attracts a steady interests in various fields of mathematics and physics, see, e.g., [7] and the reference therein. This interest grew considerably due to the recent progress in theoretical physics of $\mathcal{P} \mathcal{T}$-symmetric (pseudo-Hermitian) Hamiltonians [10,11,24]. Studies of pseudo-Hermitian operators carried out in $[22,23,27]$ shows that, even if the eigenvalues of a Hamiltonian are real, the Riesz basis property of its eigenstates might be lost.

Such kind of phenomenon is typical for $\mathcal{P} \mathcal{T}$-symmetric Hamiltonians and it gives rise to a natural problem: How to generalize the Riesz basis to be suitable to the theory of pseudo-Hermitian Hamiltonians?

One of possible generalizations was proposed by Davies [13]: the concept of tame and wild sequences. Each basis is a tame sequence. The tameness of eigenstates of non

Communicated by Mihai Putinar.

Sergiusz Kużel

kuzhel@agh.edu.pl

Alan Kamuda

kamudal@agh.edu.pl

1 AGH University of Science and Technology, 30-059 Kraków, Poland

Birkhäuser 
self-adjoint operators $H$ with a purely discrete real spectrum allows one to discover additional properties of $H$. In particular, a polynomially bounded behavior of the corresponding resolvent was established in [13, Theorem 3]. However, in major part, eigenstates of a pseudo-Hermitian Hamiltonian forms a wild system that is much more complicated for the investigation $[13,22,23]$.

Another approach to the generalization of Riesz bases is based on the rigged Hilbert spaces framework instead of the original Hilbert space [9].

In the present paper, we study generalized Riesz systems (GRS) which were originally introduced in [17,19] and then, slightly modified in $[6,8]$. In order to explain the idea of definition we note that vectors of a Riesz basis $\left\{\phi_{n}\right\}$ have the form $\phi_{n}=R e_{n}$, where $R$ is a bounded and boundedly invertible operator in a Hilbert space $\mathcal{H}$ and $\left\{e_{n}\right\}$ is an orthonormal basis (ONB) of $\mathcal{H}$. Using the polar decomposition of $R=\left|R^{*}\right| U=e^{Q / 2} U$, where $U$ is a unitary operator in $\mathcal{H}$, we conclude: a sequence $\left\{\phi_{n}\right\}$ is called a Riesz basis if there exists a bounded self-adjoint operator $Q$ in $\mathcal{H}$ and an $O N B\left\{e_{n}\right\}$ such that $\phi_{n}=e^{Q / 2} e_{n}$. This simple observation leads to:

Definition 1.1 A sequence $\left\{\phi_{n}\right\}$ is called a generalized Riesz system (GRS) if there exists a self-adjoint operator $Q$ in $\mathcal{H}$ and an $\mathrm{ONB}\left\{e_{n}\right\}$ such that $e_{n} \in \mathcal{D}\left(e^{Q / 2}\right) \cap$ $\mathcal{D}\left(e^{-Q / 2}\right)$ and $\phi_{n}=e^{Q / 2} e_{n}$.

For a GRS $\left\{\phi_{n}\right\}$, the dual GRS is determined by the formula $\left\{\psi_{n}=e^{-Q / 2} e_{n}\right\}$. Obviously, $\left\{\phi_{n}\right\}$ and $\left\{\psi_{n}\right\}$ are bi-orthogonal sequences.

Dual GRS's can be considered as a particular case of $\mathcal{G}$-quasi bases introduced by Bagarello in [4], and then analyzed in a series of papers, see, [7] and the references therein.

The main objective of the paper is further development of the GRS theory. In contrast to the standard approach [5,6,16-19], where GRS's were mainly used as auxiliary tools for the definition and investigation of manifestly non self-adjoint Hamiltonians and relevant physical operators, we consider them as a self-contained object of the basis theory $[12,15]$. Our studies are based on advanced methods of extension theory of symmetric operators, see [2,3] and Sect. 2.1.

We say that a sequence of vectors $\left\{\phi_{n}\right\}$ in a Hilbert space $\mathcal{H}$ is semi-regular if $\left\{\phi_{n}\right\}$ is minimal and complete in $\mathcal{H}$. The minimality of $\left\{\phi_{n}\right\}$ yields the existence of a bi-orthogonal sequence $\left\{\psi_{n}\right\}$. The completeness of $\left\{\phi_{n}\right\}$ guarantees the uniqueness of $\left\{\psi_{n}\right\}$. The positive symmetric operator $S$ that relates $\left\{\phi_{n}\right\}$ and $\left\{\psi_{n}\right\}$, see (2.4), plays an important role in our studies. We show that a semi-regular sequence $\left\{\phi_{n}\right\}$ is a GRS if and only if the Friedrichs extension $A_{F}$ of $S$ is a positive operator (Theorem 2.5). It should be noted that our definition of semi-regular sequences is more general than the definition given by Inoue in [17]. We discuss this point in Remark 2.6.

Theorem 2.5 allows one to explain the nonuniqueness of self-adjoint operators $Q$ in the definition of GRS (Sect. 2.3). Further we show that each semi-regular sequence $\left\{\phi_{n}\right\}$ with the property of being Bessel sequence has to be a GRS and we characterize this important case in terms of $Q$ (Theorem 2.13). The Olevskii's result [25, Theorem 1] allows one to establish a relationship between essential spectra of self-adjoint operators $Q$ and conditional bounded bases (Proposition 2.14).

The end of Sect. 2 is devoted to an important particular case (which fits well the specific of $\mathcal{P} \mathcal{T}$-symmetric Hamiltonians) where a semi-regular sequence $\left\{\phi_{n}\right\}$ is $J$ - 
orthonormal. Following [8], we define $J$-orthonormal sequences of the first/second type and discuss advantages of first type sequences. In particular, eigenstates of the shifted harmonic oscillator form a $J$-orthonormal sequence of the first type and it seems natural to suppose that eigenstates of a $\mathcal{P} \mathcal{T}$-symmetric Hamiltonian with unbroken $\mathcal{P} \mathcal{T}$-symmetry [10, p. 41] form a first type sequence. In Sect. 3.3 we describe a general method which allows one to construct of the first/second type sequences.

Throughout the paper, $\mathcal{D}(A), \mathcal{R}(A)$, and ker $A$ denote the domain, the range, and the null-space of a linear operator $A$, respectively. The symbol $\left\{u_{n}\right\}$ means the collection of vectors $u_{n}$ parametrized by a set $\mathcal{I}$ of integers. Usually, $\mathcal{I}=\mathbb{N}$.

\section{General Theory of GRS}

\subsection{Preliminaries}

Here, all necessary results of extension theory of symmetric operators are presented in a form convenient for our exposition. The articles [1-3] and [26, Chap. 10] are recommended as complementary reading on the subject.

Let $\mathcal{H}$ be a complex Hilbert space with inner product $(\cdot, \cdot)$ linear in the first argument. An operator $A$ is called positive [nonnegative] if $(A f, f)>0[(A f, f) \geq 0]$ for nonzero $f \in \mathcal{D}(A)$.

Let $A$ and $B$ be nonnegative self-adjoint operators. We say that $A$ is greater or equal $B$, i.e., $A \geq B$ if

$$
\mathcal{D}\left(A^{1 / 2}\right) \subseteq \mathcal{D}\left(B^{1 / 2}\right) \text { and }\left\|A^{1 / 2} f\right\| \geq\left\|B^{1 / 2} f\right\|, \quad f \in \mathcal{D}\left(A^{1 / 2}\right)
$$

The next technical result follows from (2.1) (see [26, Corollary 10.12]).

Lemma 2.1 If $A \geq B$ and $B$ is positive, then $A$ is also positive.

Let $S$ be a nonnegative densely defined operator in $\mathcal{H}$. M. Krein established that the set of nonnegative self-adjoint extensions $\{A\}$ of $S$ can be ordered as follows [3, Theorem 3.5]: $A_{F} \geq A \geq A_{K}$, where the greatest self-adjoint extension $A_{F}$ is called the Friedrichs extension, while the smallest one $A_{K}$ is called the Krein-von Neumann extension.

The extensions $A_{K}$ and $A_{F}$ are examples of extremal extensions. We recall [2] that a nonnegative self-adjoint extension $A$ of $S$ is called extremal if

$$
\inf _{f \in \mathcal{D}(S)}(A(\phi-f),(\phi-f))=0 \text { for all } \phi \in \mathcal{D}(A)
$$

If a nonnegative self-adjoint extension $A$ of $S$ is positive, we can set $A=e^{-Q}$, where $Q=-\ln A$ is a self-adjoint operator in $\mathcal{H}$ and define the new Hilbert space $\mathcal{H}_{-} Q$ as the completion of $\mathcal{D}(A)=\mathcal{D}\left(e^{-Q}\right)$ with respect to the new inner product

$$
(f, g)_{-Q}:=\left(e^{-Q} f, g\right)=\left(e^{-Q / 2} f, e^{-Q / 2} g\right), \quad f, g \in \mathcal{D}\left(e^{-Q}\right)
$$


Rewriting (2.2) as

$$
\inf _{f \in \mathcal{D}(S)}(\phi-f, \phi-f)_{-Q}=\inf _{f \in \mathcal{D}(S)}\|\phi-f\|_{-Q}^{2}=0 \text { for all } \phi \in \mathcal{D}\left(e^{-Q}\right)
$$

we obtain the following.

Lemma 2.2 A positive self-adjoint extension $A=e^{-Q}$ of $S$ is an extremal extension if and only if $\mathcal{D}(S)$ is a dense set in $\mathcal{H}_{-Q}$.

A non-negative, non-densely defined symmetric operator $S$ admits self-adjoint extensions, but not necessarily non-negative ones. The well-known Ando-Nishio result is [1, Theorem 1]:

Lemma 2.3 A closed non-negative symmetric operator $S$ admits a non-negative selfadjoint extension if and only if it is positively closable, i.e., if the relations

$$
\lim _{n \rightarrow \infty}\left(S f_{n}, f_{n}\right)=0 \quad \text { and } \quad \lim _{n \rightarrow \infty} S f_{n}=g
$$

implies $g=0$.

Lemma 2.3 is obvious for densely defined operators because each densely defined operator $S$ is positively closable [1, p. 67]. Another useful result follows from [1, Corollary 4]:

Lemma 2.4 Let $S$ be a closed densely defined positive operator. Then the Friedrichs extension $A_{F}$ of $S$ is positive if and only if $S^{-1}$ admits a non-negative self-adjoint extension.

\subsection{Conditions of Being GRS}

Let $\left\{\phi_{n}\right\}$ be a GRS. In view of Definition 1.1, the sequence $\left\{\psi_{n}=e^{-Q / 2} e_{n}\right\}$ is well defined and it is a bi-orthogonal sequence for $\left\{\phi_{n}=e^{Q / 2} e_{n}\right\}$. Obviously, $\left\{\psi_{n}\right\}$ is a GRS which we call a dual GRS.

The existence of a bi-orthogonal sequence means that each GRS $\left\{\phi_{n}\right\}$ has to be a minimal sequence, i.e., $\phi_{j} \notin \overline{\operatorname{span}}\left\{\phi_{k}\right\}_{k \neq j}$ [12, Lemma 3.3.1]. However, not each minimal sequence is a GRS.

We say that a minimal sequence $\left\{\phi_{n}\right\}$ is semi-regular if $\left\{\phi_{n}\right\}$ is complete in $\mathcal{H}$ and regular if its bi-orthogonal sequence $\left\{\psi_{n}\right\}$ is also complete. For a semi-regular sequence $\left\{\phi_{n}\right\}$ the corresponding bi-orthogonal sequence $\left\{\psi_{n}\right\}$ is determined uniquely.

Let $\left\{\phi_{n}\right\}$ be a minimal sequence. Then there exists a bi-orthogonal sequence $\left\{\psi_{n}\right\}$ and we can consider an operator $S$ defined initially on

$$
S \phi_{n}=\psi_{n},
$$

and extended on $\mathcal{D}(S)=\operatorname{span}\left\{\phi_{n}\right\}$ by the linearity. By the construction,

$$
(S f, f)=\sum_{n=1}^{k} \sum_{m=1}^{k} c_{n} \bar{c}_{m}\left(\psi_{n}, \phi_{m}\right)=\sum_{n=1}^{k}\left|c_{n}\right|^{2} \text { for all } f=\sum_{n=1}^{k} c_{n} \phi_{n} \in \mathcal{D}(S) .
$$


Therefore, $S$ is a positive operator. For a semi-regular sequence $\left\{\phi_{n}\right\}$, the operator $S$ is densely defined and the Friedrichs extension $A_{F}$ of $S$ exists.

Theorem 2.5 Let $\left\{\phi_{n}\right\}$ be a semi-regular sequence. The following are equivalent:

(i) $\left\{\phi_{n}\right\}$ is a GRS;

(ii) the Friedrichs extension $A_{F}$ of $S$ is a positive operator;

(iii) the closure $\bar{S}$ of $S$ is a positive operator and the relations

$$
\lim _{n \rightarrow \infty}\left(\bar{S} f_{n}, f_{n}\right)=0 \quad \text { and } \quad \lim _{n \rightarrow \infty} f_{n}=g
$$

imply that $g=0$;

(iv) the set $D(\phi)=\left\{f \in \mathcal{H}: \sum_{n=1}^{\infty}\left|\left(f, \phi_{n}\right)\right|^{2}<\infty\right\}$ is dense in $\mathcal{H}$.

Proof (i) $\rightarrow$ (ii) If $\left\{\phi_{n}\right\}$ is a GRS, then $e^{-Q} \phi_{n}=e^{-Q / 2} e_{n}=\psi_{n}$. In view of (2.4), $e^{-Q}$ is a positive self-adjoint extension of $S$ and $A_{F} \geq e^{-Q}$ since the Friedrichs extension is the greatest nonnegative self-adjoint extension of $S$. By virtue of Lemma 2.1, $A_{F}$ is positive.

(ii) $\rightarrow$ (i) The positivity of $A_{F}$ means that $A_{F}=e^{-Q}$, where $Q$ is a self-adjoint operator in $\mathcal{H}$. Denote $e_{n}=e^{-Q / 2} \phi_{n}$. Due to (2.4), $e_{n}=e^{Q / 2} \psi_{n}$. Therefore, $e_{n} \in$ $\mathcal{D}\left(e^{Q / 2}\right) \cap \mathcal{D}\left(e^{-Q / 2}\right)$ and $\left(e_{n}, e_{m}\right)=\left(e^{-Q / 2} \phi_{n}, e^{Q / 2} \psi_{m}\right)=\left(\phi_{n}, \psi_{m}\right)=\delta_{n m}$.

The orthonormal sequence $\left\{e_{n}\right\}$ turns out to be an ONB if $\left\{e_{n}\right\}$ is complete in $\mathcal{H}$. Assume that $\gamma$ is orthogonal to $\left\{e_{n}\right\}$ in $\mathcal{H}$. Then there exists a sequence $\left\{f_{m}\right\}$ $\left(f_{m} \in \mathcal{D}\left(e^{-Q}\right)\right)$ such that $e^{-Q / 2} f_{m} \rightarrow \gamma$ in $\mathcal{H}$ (because $e^{-Q / 2} \mathcal{D}\left(e^{-Q}\right)$ is a dense set in $\mathcal{H})$. In this case, due to (2.3), $\left\{f_{m}\right\}$ is a Cauchy sequence in $\mathcal{H}_{-} Q$ and therefore, $f_{m}$ tends to some $f \in \mathcal{H}_{-}$. This means that

$$
0=\left(\gamma, e_{n}\right)=\lim _{m \rightarrow \infty}\left(e^{-Q / 2} f_{m}, e_{n}\right)=\lim _{m \rightarrow \infty}\left(f_{m}, \phi_{n}\right)_{-Q}=\left(f, \phi_{n}\right)_{-Q}
$$

By Lemma 2.2, the set $\mathcal{D}(S)=\operatorname{span}\left\{\phi_{n}\right\}$ is dense in the Hilbert space $\mathcal{H}_{-Q}$. Due to (2.6), $f=0$ that means $\lim _{m \rightarrow \infty}\left\|f_{m}\right\|_{-Q}=\lim _{m \rightarrow \infty}\left\|e^{-Q / 2} f_{m}\right\|=0$ and hence, $\gamma=0$. Thus, $\left\{e_{n}\right\}$ is complete in $\mathcal{H}$ and $\left\{e_{n}\right\}$ is an ONB of $\mathcal{H}$.

The implication (ii) $\rightarrow$ (iii) is obvious.

(iii) $\rightarrow$ (ii) The operator $\bar{S}$ has the inverse $\bar{S}^{-1}$ since $\bar{S}$ is positive. The operator $\bar{S}^{-1}$ is closed and (2.5) can be rewritten as follows:

$$
\lim _{n \rightarrow \infty}\left(g_{n}, \bar{S}^{-1} g_{n}\right)=0 \quad \text { and } \quad \lim _{n \rightarrow \infty} \bar{S}^{-1} g_{n}=g \quad\left(g_{n}=\bar{S} f_{n}\right)
$$

This means that $\bar{S}^{-1}$ is positively closable. Hence, it admits a non-negative self-adjoint extension (Lemma 2.3) Applying Lemma 2.4 we complete the proof of (iii) $\rightarrow$ (ii).

The implication (i) $\rightarrow$ (iv) follows from the fact that $\mathcal{R}\left(e^{-Q / 2}\right) \subset D(\phi)$ since, for all $u \in \mathcal{D}\left(e^{-Q / 2}\right)$,

$$
\sum_{n=1}^{\infty}\left|\left(e^{-Q / 2} u, \phi_{n}\right)\right|^{2}=\sum_{n=1}^{\infty}\left|\left(u, e^{-Q / 2} \phi_{n}\right)\right|^{2}=\sum_{n=1}^{\infty}\left|\left(u, e_{n}\right)\right|^{2}=\|u\|^{2}<\infty .
$$


(iv) $\rightarrow$ (ii) Following [17] we consider the densely defined operators

$$
T_{e \phi} f=\sum_{n=1}^{\infty}\left(f, \phi_{n}\right) e_{n}, \quad \mathcal{D}\left(T_{e \phi}\right)=D(\phi), \quad T_{\phi e} g=\sum_{n=1}^{\infty}\left(g, e_{n}\right) \phi_{n},
$$

$\mathcal{D}\left(T_{\phi e}\right)=\left\{g \in \mathcal{H}:\right.$ the series $\sum_{n=1}^{\infty}\left(g, e_{n}\right) \phi_{n}$ converges in $\left.\mathcal{H}\right\}$.

It is easy to see that $T_{e \phi}$ is a closable operator and $\operatorname{ker} \bar{T}_{e \phi}=\{0\}$, where $\bar{T}_{e \phi}$ is the closure of $T_{e \phi}$. Therefore, the operator $B=T_{e \phi}^{*} \bar{T}_{e \phi}$ is positive self-adjoint in $\mathcal{H}$. Here $T_{e \phi}^{*} \supset T_{\phi e}$, since $\left(T_{e \phi} f, g\right)=\left(f, T_{\phi e} g\right)$ for $f \in \mathcal{D}\left(T_{e \phi}\right)$ and $g \in \mathcal{D}\left(T_{\phi e}\right)$. Taking into account that $T_{e \phi} \psi_{n}=e_{n}$ and $T_{\phi e} e_{n}=\phi_{n}$, we obtain $B \psi_{n}=\phi_{n}$. Hence, $B$ is a positive self-adjoint extension of $S^{-1}$. This means that $A=B^{-1}$ is a positive self-adjoint extension of $S$ and, in view of Lemma 2.1, the Friedrichs extension $A_{F}$ of $S$ is also a positive operator.

Remark 2.6 The item (iv) of Theorem 2.5 was proved in [17] [item (2) of Theorem 3.4]. The definition of a semi-regular sequence $\left\{\phi_{n}\right\}$ given in [17] (Definition 3.3) involves the condition (iv) as an additional assumption. For this reason each Inoue's semi-regular sequence $\left\{\phi_{n}\right\}$ has to be a GRS.

It is worth mentioning that the example of semi-regular sequence $\left\{\phi_{n}\right\}$ given in [17] after Definition 3.3 may be misleading because $\left\{\phi_{n}\right\}$ is not a semi-regular sequence in the sense of [17] and it cannot be a GRS. Indeed, the sequence $\left\{\phi_{n}\right\}$ is defined as $\phi_{n}=\mathrm{e}_{n}+\mathrm{e}_{0}, n=1,2 \ldots$, where $\left\{\mathrm{e}_{n}\right\}_{n=0}^{\infty}$ is an ONB of $\mathcal{H}$. It is easy to see that $\left\{\phi_{n}\right\}$ is complete in $\mathcal{H}$ and $\left\{\phi_{n}\right\}$ is minimal (since there exists a bi-orthogonal sequence $\left\{\psi_{n}=\right.$ $\left.\left.\mathrm{e}_{n}\right\}_{n=1}^{\infty}\right)$. If $f=\sum_{n=0}^{\infty} f_{n} e_{n}$ belongs to $D(\phi)$, then $\sum_{n=1}^{\infty}\left|\left(f, \phi_{n}\right)\right|^{2}=\sum_{n=1}^{\infty} \mid f_{n}+$ $\left.f_{0}\right|^{2}<\infty$. The last inequality is possible only for the case $f_{0}=0$. Therefore, the vector $\mathrm{e}_{0}$ is orthogonal to $D(\phi)$. A generalization of this example is considered in Sect. 3.1.

\section{Corollary 2.7 A regular sequence is a GRS.}

Proof Let $\left\{\phi_{n}\right\}$ be a regular sequence. Its regularity means that $\mathcal{R}(S)$ is a dense set in $\mathcal{H}$. The latter means that each nonnegative self-adjoint extension $A$ of $S$ must be positive. In particular, the Friedrichs extension $A_{F}$ is positive. By Theorem $2.5,\left\{\phi_{n}\right\}$ is a GRS.

A shifting of the orthonormal Hermite functions $e_{n}(x)$ in the complex plane gives rise to regular sequences in $L_{2}(\mathbb{R})$. In particular, eigenfunctions of the shifted harmonic oscillator $\left\{\phi_{n}(x)=e_{n}(x+i a)\right\}$ form a regular sequence and $\phi_{n}=e^{Q / 2} e_{n}$, where $Q=2 a i \frac{d}{d x}$ is an unbounded self-adjoint operator in $L_{2}(\mathbb{R})$ [8, Subsection IV.1]. We refer $[16,19]$ to the relationship between general regular sequences and some physical operators.

Remark 2.8 Theorem 2.5 can be generalized to the case of non-complete minimal sequence $\left\{\phi_{n}\right\}$ such that its bi-orthogonal $\left\{\psi_{n}\right\}$ is also non-complete. In this case, $S$ is a non-densely defined positive symmetric operator in $\mathcal{H}$. We should suppose the existence of a positive self-adjoint extension $A$ of $S$. Similarly to the proof of Theorem 2.5 we set $A=e^{-Q}$ and determine the orthonormal system $\left\{e_{n}\right\}$ in $\mathcal{H}$. By virtue of 
(2.6), the completeness of $\left\{e_{n}\right\}$ in $\mathcal{H}$ is equivalent to the completeness of $\left\{\phi_{n}\right\}$ in $\mathcal{H}_{-}$. This means that $\left\{e_{n}\right\}$ is an ONB of $\mathcal{H}$ if and only if $\left\{\phi_{n}\right\}$ is complete in $\mathcal{H}_{-Q}$. Summing up: Let $\left\{\phi_{n}\right\}$ be a minimal sequence and let $\left\{\psi_{n}\right\}$ be its bi-orthogonal sequence. These sequences are GRS if and only if there exists a positive self-adjoint extension $A=e^{-Q}$ of $S$ such that $\left\{\phi_{n}\right\}$ is a complete set in the Hilbert space $\mathcal{H}_{-Q}$.

Another approach to the study of non-complete minimal sequences can be found in [6].

\subsection{The Uniqueness of $Q$ in the Definition of GRS}

Let $\left\{\phi_{n}\right\}$ be a basis in $\mathcal{H}$. Then $\left\{\phi_{n}\right\}$ is a regular sequence because its bi-orthogonal sequence $\left\{\psi_{n}\right\}$ has to be a basis [15, Corollary 5.22]. By Corollary 2.7, $\left\{\phi_{n}\right\}$ is a GRS, i.e., $\phi_{n}=e^{Q / 2} e_{n}$. Moreover, by [8, Proposition II.9], the pair $\left(Q,\left\{e_{n}\right\}\right)$ in Definition 1.1 is determined uniquely for every basis $\left\{\phi_{n}\right\}$. For this reason, a natural question arise: is the pair $\left(Q,\left\{e_{n}\right\}\right)$ determined uniquely for a given $G R S\left\{\phi_{n}\right\}$ ?

The choice of the Friedrichs extension $A_{F}=e^{-Q}$ of $S$ in the proof of Theorem 2.5 was related to the fact that $\mathcal{D}(S)$ must be dense in the Hilbert space $\mathcal{H}_{-} Q$ (that, in view of (2.6), is equivalent to the completeness of $\left\{e_{n}\right\}$ in $\mathcal{H}$ ). Due to Lemma 2.2, each positive extremal extension $A=e^{-Q}$ can be used instead of $A_{F}$ in the proof of Theorem 2.5. This observation leads to the following result ([8, Proposition II.10]):

Proposition 2.9 Let a semi-regular sequence $\left\{\phi_{n}\right\}$ be a GRS. Then a self-adjoint operator $Q$ and an $O N B\left\{e_{n}\right\}$ are determined uniquely in the formula $\phi_{n}=e^{Q / 2} e_{n}$ if and only the symmetric operator $S$ in (2.4) has a unique positive extremal extension.

Remark 2.10 The above mentioned unique positive extremal extension coincides with the Friedrichs extension $A_{F}$. Indeed, the existence of another positive extension $A \neq$ $A_{F}$ means that $A_{F}$ is also positive (Lemma 2.1). Due to the uniqueness of positive extension, we get $A=A_{F}$.

\subsection{Bases and Bessel Sequences}

Various classes of GRS's can be easily characterized in terms of spectral properties of the corresponding self-adjoint operators $Q$.

We recall that a semi-regular sequence $\left\{\phi_{n}\right\}$ is called a Riesz basis if there exists $0<a \leq b$ such that ${ }^{1}$

$$
a\|f\|^{2} \leq \sum_{n}\left|\left(f, \phi_{n}\right)\right|^{2} \leq b\|f\|^{2} \text { for all } f \in \mathcal{H} .
$$

Proposition 2.11 The following are equivalent:

(i) a sequence $\left\{\phi_{n}\right\}$ is a Riesz basis with bounds $0<a \leq b$;

(ii) $\left\{\phi_{n}\right\}$ is a GRS, i.e., $\phi_{n}=e^{Q / 2} e_{n}$, where $Q$ is a bounded self-adjoint operator such that $\sigma(Q) \subset[\ln a, \ln b]$.

\footnotetext{
${ }^{1}$ We refer [15, Theorems 7.13, 8.32] to the equivalent definitions of Riesz basis.
} 
Proof If $\left\{\phi_{n}\right\}$ is a Riesz basis, then $\phi_{n}=e^{Q / 2} e_{n}$, where $Q$ is a bounded operator (see Sect. 1). The substitution of $\phi_{n}=e^{Q / 2} e_{n}$ into (2.7) gives

$$
a(f, f)=a\|f\|^{2} \leq \sum_{n}\left|\left(e^{Q / 2} f, e_{n}\right)\right|^{2} \leq\left\|e^{Q / 2} f\right\|^{2}=\left(e^{Q} f, f\right) \leq b\|f\|^{2}=b(f, f) .
$$

Therefore, $a I \leq e^{Q} \leq b I$ that justifies (i) $\rightarrow$ (ii). The converse statement is obvious.

Lemma 2.12 Let $Q$ be a self-adjoint operator such that $\sigma(Q) \subset(-\infty, \ln b]$ and let $\left\{e_{n}\right\}$ be an arbitrary $O N B$ of $\mathcal{H}$. Then the sequence $\left\{\phi_{n}=e^{Q / 2} e_{n}\right\}$ is a GRS with the $\operatorname{pair}\left(Q,\left\{e_{n}\right\}\right)$.

Proof Since $\sigma(Q) \subset(-\infty, \ln b]$, the self-adjoint operator $e^{Q / 2}$ is bounded. Hence, the elements $\phi_{n}=e^{Q / 2} e_{n}$ are well-defined. If $\gamma$ is orthogonal to $\left\{\phi_{n}\right\}$, then $0=\left(\gamma, e^{Q / 2} e_{n}\right)=\left(e^{Q / 2} \gamma, e_{n}\right)$ implies that $e^{Q / 2} \gamma=\gamma=0$. Therefore $\left\{\phi_{n}\right\}$ is semi-regular and its bi-orthogonal sequence $\left\{\psi_{n}\right\}$ is defined uniquely. According to Definition 1.1, it is sufficient to show that $e_{n} \in \mathcal{D}\left(e^{-Q / 2}\right)$ and $\psi_{n}=e^{-Q / 2} e_{n}$. By virtue of the relation $\delta_{m n}=\left(\phi_{m}, \psi_{n}\right)=\left(e^{Q / 2} e_{m}, \psi_{n}\right)=\left(e_{m}, e^{Q / 2} \psi_{n}\right)$ we obtain that $e^{Q / 2} \psi_{n}=e_{n}$. The last relation means that $e_{n} \in \mathcal{D}\left(e^{-Q / 2}\right)$ and $\psi_{n}=e^{-Q / 2} e_{n}$.

A sequence $\left\{\phi_{n}\right\}$ is called $a$ Bessel sequence if there exists $b>0$ such that

$$
\sum_{n}\left|\left(f, \phi_{n}\right)\right|^{2} \leq b\|f\|^{2} \text { for all } f \in \mathcal{H}
$$

Theorem 2.13 The following are equivalent:

(i) a semi-regular sequence $\left\{\phi_{n}\right\}$ is a Bessel sequence;

(ii) $\left\{\phi_{n}\right\}$ is a GRS, i.e., $\phi_{n}=e^{Q / 2} e_{n}$, where $Q$ is a self-adjoint operator such that $\sigma(Q) \subset(-\infty, \ln b]$.

Proof (i) $\rightarrow$ (ii) If $\left\{\phi_{n}\right\}$ is a Bessel sequence, then the synthesis operator $R\left\{c_{n}\right\}=$ $\sum c_{n} \phi_{n}$ defines a bounded operator which maps $l^{2}(\mathbb{N})$ into $\mathcal{H}[15, \mathrm{p} .190]$. The minimality of $\left\{\phi_{n}\right\}$ implies that $\left\{\phi_{n}\right\}$ is $\omega$-independent [15, p. 156]. The latter means that the series $\sum c_{n} \phi_{n}$ converges and equal 0 only when $c_{n}=0$. Therefore, ker $R=\{0\}$.

Let $\left\{\delta_{n}\right\}$ be the canonical basis of $l^{2}(\mathbb{N})$. Then $R \delta_{n}=\phi_{n}$. Identifying $\left\{\delta_{n}\right\}$ with an ONB $\left\{\tilde{e}_{n}\right\}$ of $\mathcal{H}$ we obtain a bounded operator ${ }^{2} R$ in $\mathcal{H}$ such $R \tilde{e}_{n}=\phi_{n}$. The polar decomposition of $R$ is $R=\left|R^{*}\right| U$, where $\left|R^{*}\right|=\sqrt{R R^{*}}$ and $U$ is an isometric operator mapping the closure of $\mathcal{R}\left(\sqrt{R^{*} R}\right)$ onto the closure of $\mathcal{R}(R)$ [21, Chapter VI, Subsect. 2.7]. We remark that $\mathcal{R}\left(\sqrt{R^{*} R}\right)$ and $\mathcal{R}(R)$ are dense sets in $\mathcal{H}$, since, respectively, $\operatorname{ker} \sqrt{R^{*} R}=\operatorname{ker} R=\{0\}$ and $\left\{\phi_{n}\right\}$ is a complete set in $\mathcal{H}$. Therefore, $U$ is a unitary operator in $\mathcal{H}$. Moreover, $\left|R^{*}\right|$ is a positive bounded self-adjoint operator (since $\operatorname{ker}\left|R^{*}\right|=\operatorname{ker} R=\{0\}$ ). The positivity of $\left|R^{*}\right|$ leads to the formula

\footnotetext{
${ }^{2}$ We keep the same notation $R$ for the operator in $\mathcal{H}$.
} 
$\left|R^{*}\right|=e^{Q / 2}$, where $Q$ is a self-adjoint operator in $\mathcal{H}$. Denote $e_{n}=U \tilde{e}_{n}$. Obviously, $\left\{e_{n}\right\}$ is an ONB of $\mathcal{H}$ and

$$
\phi_{n}=R \tilde{e}_{n}=\left|R^{*}\right| U \tilde{e}_{n}=e^{Q / 2} U \tilde{e}_{n}=e^{Q / 2} e_{n} .
$$

After the substitution of $\phi_{n}=e^{Q / 2} e_{n}$ into (2.8),

$$
\sum_{n} \mid\left(f,\left.e^{Q / 2} e_{n}\right|^{2}=\sum_{n} \mid\left(e^{Q / 2} f,\left.e_{n}\right|^{2}=\left\|e^{Q / 2} f\right\|^{2}=\left(e^{Q} f, f\right) \leq b(f, f) .\right.\right.
$$

The obtained inequality leads to the conclusion that $\sigma(Q) \subset(-\infty, \ln b]$. Applying Lemma 2.12 we complete the proof of (i) $\rightarrow$ (ii).

(ii) $\rightarrow$ (i) In view of Lemma 2.12, $\left\{\phi_{n}\right\}$ is a semi-regular sequence. The operator $e^{Q}$ is bounded and $\left\|e^{Q}\right\| \leq b($ since $\sigma(Q) \subset(-\infty, \ln b])$. Hence,

$$
\sum_{n}\left|\left(f, \phi_{n}\right)\right|^{2}=\sum_{n} \mid\left(e^{Q / 2} f,\left.e_{n}\right|^{2}=\left\|e^{Q / 2} f\right\|^{2}=\left(e^{Q} f, f\right) \leq b\|f\|^{2}\right.
$$

that completes the proof.

A sequence $\left\{\phi_{n}\right\}$ is called bounded if $0<a \leq\left\|\phi_{n}\right\| \leq b$ for all $n$. A basis $\left\{\phi_{n}\right\}$ is called conditional if its property of being basis depends on the permutation of elements $\phi_{n}$.

Proposition 2.14 Let $Q$ be a self-adjoint operator in $\mathcal{H}$ such that $\sigma(Q) \subset(-\infty, \ln b]$. The following are equivalent:

(i) there exists an $O N B\left\{e_{n}\right\}$ of $\mathcal{H}$ such that the sequence $\left\{\phi_{n}=e^{Q / 2} e_{n}\right\}$ is a conditional bounded basis;

(ii) there exists $\beta<0$ such that each interval $[(n+1) \beta, n \beta](n=0,1, \ldots)$ includes at least one point of essential spectrum of $Q$.

Proof Applying [25, Theorem 1] to the positive bounded operator $e^{Q / 2}$ and taking into account properties of an essential spectrum [26, Proposition 8.11] we arrive at the conclusion that the item (i) is equivalent to the existence of $0<q<1$ such that the essential spectrum of $e^{Q / 2}$ has a non-zero interaction with each interval $\left[q^{n+1}, q^{n}\right]$. Since $Q=2 \ln e^{Q / 2}$, the later statement is equivalent to (ii) with $\beta=2 \ln q$.

Let $\mathcal{H}=L_{2}(-\pi, \pi)$ and $Q$ is an operator of multiplication by $\alpha \ln |x|(0<\alpha)$ in $\mathcal{H}$. Obviously, $Q$ is self-adjoint, its spectrum coincides with $\left(-\infty, \ln \pi^{\alpha}\right]$ and it is essential. By Proposition 2.14, there exists an ONB $\left\{e_{n}\right\}$ of $L_{2}(-\pi, \pi)$ such that $\left\{\phi_{n}=e^{Q / 2} e_{n}\right\}$ is a conditional bounded basis. In view of the Babenko example [15, Example 5.13], for $0<\alpha<\frac{1}{2}$, the corresponding ONB can be chosen as $\left\{e_{n}=\frac{1}{\sqrt{2 \pi}} e^{i n x}\right\}_{-\infty}^{\infty}$. 


\subsection{J-Orthonormal Sequences and GRS}

Let $J$ be a bounded self-adjoint operator in a Hilbert space $\mathcal{H}$ such that $J^{2}=I$. The Hilbert space $\mathcal{H}$ equipped with the indefinite inner product $[\cdot, \cdot]:=(\mathrm{J} \cdot, \cdot)$ is called a Krein space.

A sequence $\left\{\phi_{n}\right\}$ is called $J$-orthonormal if $\left|\left[\phi_{n}, \phi_{m}\right]\right|=\delta_{n m}$.

Each $J$-orthonormal sequence $\left\{\phi_{n}\right\}$ is minimal since its bi-orthogonal one is determined as

$$
\psi_{n}=\left[\phi_{n}, \phi_{n}\right] J \phi_{n}
$$

In view of (2.9), the positive symmetric operator $S$ in (2.4) acts as $S \phi_{n}=\left[\phi_{n}, \phi_{n}\right] J \phi_{n}$.

Proposition 2.15 Let $\left\{\phi_{n}\right\}$ be a complete J-orthonormal sequence. Then $\left\{\phi_{n}\right\}$ is a Bessel sequence if and only if $\left\{\phi_{n}\right\}$ is a Riesz basis.

Proof Let us assume that $\left\{\phi_{n}\right\}$ is a Bessel sequence. Then $\left\{\psi_{n}\right\}$ is also a Bessel sequence. Indeed, substituting $J f$ instead of $f$ into (2.8) and using (2.9), we obtain

$$
\sum_{n}\left|\left(J f, \phi_{n}\right)\right|^{2}=\sum_{n}\left|\left(f, J \phi_{n}\right)\right|^{2}=\sum_{n}\left|\left(f, \psi_{n}\right)\right|^{2} \leq b\|J f\|^{2}=b\|f\|^{2} .
$$

Due to Theorem 2.13, $\left\{\phi_{n}\right\}$ is a GRS and $\phi_{n}=e^{Q / 2} e_{n}$, where $\sigma(Q) \subset(-\infty, \ln b]$. Since $\left\{\psi_{n}\right\}$ is also a Bessel sequence, applying Theorem 2.13 again we obtain $\sigma(-Q) \subset(-\infty, \ln b]$ or $\sigma(Q) \subset[-\ln b, \infty)$. Therefore, $\sigma(Q) \subset[\ln a, \ln b]$, where $a=1 / b$. In view of Proposition 2.11, $\left\{\phi_{n}\right\}$ is a Riesz basis. The inverse statement is obvious.

If $\left\{\phi_{n}\right\}$ is complete in $\mathcal{H}$, then $\left\{\psi_{n}\right\}$ in (2.9) is complete too. Therefore, $\left\{\phi_{n}\right\}$ is regular and, by Corollary $2.7,\left\{\phi_{n}\right\}$ is a GRS. Thus, each complete J-orthonormal sequence is a GRS.

It follows from the proof of Corollary 2.7 that each extremal extension $A$ of $S$ is positive. Therefore, the corresponding operator $Q=-\ln A$ in Definition 1.1 can be determined by every extremal extension $A$. If $Q$ is determined uniquely, then [8, Theorem III.3]:

$$
J Q=-Q J
$$

However, if $Q$ is not determined uniquely, not each $Q=-\ln A$ satisfies (2.10). In particular, as follows from [20], the operator $Q$ that corresponds to the Friedrichs extension $A_{F}$ does not satisfy (2.10). Moreover, there exist complete $J$-orthonormal sequences for which no operators $Q$ satisfying (2.10) can be found.

We say that a complete J-orthonormal sequence $\left\{\phi_{n}\right\}$ is of the first type if there exists a self-adjoint operator $Q$ in Definition 1.1 such that (2.10) holds. Otherwise, $\left\{\phi_{n}\right\}$ is of the second type.

$J$-orthonormal bases are examples of the first type sequences. The next statement was proved in [20], where the notation "quasi-bases" was used for the first type sequences. 
Proposition 2.16 The following are equivalent:

(i) a complete J-orthonormal sequence $\left\{\phi_{n}\right\}$ is of the first type;

(ii) the sequence $\left\{\phi_{n}\right\}$ is regular and the corresponding pair $\left(Q,\left\{e_{n}\right\}\right)$ in Definition 1.1 can be chosen as follows: $Q$ satisfies (2.10) and $e_{n}$ are eigenfunctions of $J$, i.e., $J e_{n}=e_{n}$ or $J e_{n}=-e_{n}$.

In what follows, by considering a first type sequence $\left\{\phi_{n}=e^{Q / 2} e_{n}\right\}$, we assume that the pair $\left(Q,\left\{e_{n}\right\}\right)$ satisfies conditions (ii) of Proposition 2.16. A detailed analysis of the first/second type sequences can be found in [8]. We just mention that a first type sequence $\left\{\psi_{n}=e^{Q / 2} e_{n}\right\}$ generates a $\mathcal{C}$-symmetry operator ${ }^{3} \mathcal{C}=e^{Q} J$ with the same operator $Q$. The latter allows one to construct the new Hilbert space $\mathcal{H}_{-} Q$

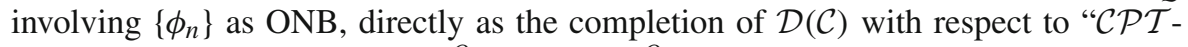
norm": $(\cdot, \cdot)_{-Q}=[\mathcal{C} \cdot, \cdot]=\left(J e^{Q} J \cdot, \cdot\right)=\left(e^{-Q \cdot, \cdot}\right)$.

For a second type sequence, the inner product $(\cdot, \cdot)_{-}$defined by $(2.3)$ cannot be expressed via $[\cdot, \cdot]$ and one should apply much more efforts for the precise definition of $(\cdot, \cdot)-Q \cdot$

\section{Examples}

\subsection{A Semi-regular Sequence that Cannot be a GRS}

Let $\left\{\mathrm{e}_{n}\right\}_{n=0}^{\infty}$ be an ONB of $\mathcal{H}$. Denote

$$
\phi_{n}=\frac{1}{n^{\beta}} \mathrm{e}_{n}+\frac{1}{n^{\alpha}} \mathrm{e}_{0}, \quad \alpha, \beta \in \mathbb{R}, \quad n=1,2, \ldots
$$

The sequence $\left\{\phi_{n}\right\}_{n=1}^{\infty}$ is minimal since $\left\{\psi_{n}=n^{\beta} \mathrm{e}_{n}\right\}_{n=1}^{\infty}$ is bi-orthogonal to $\left\{\phi_{n}\right\}$. It is easy to see that $\left\{\phi_{n}\right\}$ is complete in $\mathcal{H}$ if and only if $\alpha-\beta \leq \frac{1}{2}$. The last relation determines admissible parameters $\alpha, \beta$ for which $\left\{\phi_{n}\right\}$ is a semi-regular sequence. Throughout Sect. 3.1 we suppose that this inequality holds.

In view of (2.4), $S\left(\frac{1}{n^{\beta}} \mathrm{e}_{n}+\frac{1}{n^{\alpha}} \mathrm{e}_{0}\right)=n^{\beta} \mathrm{e}_{n}$ and the operator $S$ can be described as:

$$
S f=\sum_{n=1}^{k} n^{2 \beta} c_{n} \mathrm{e}_{n} \text { for all } f=\sum_{n=1}^{k} c_{n} \mathrm{e}_{n}+\left(\sum_{n=1}^{k} \frac{n^{\beta} c_{n}}{n^{\alpha}}\right) \mathrm{e}_{0} \in \mathcal{D}(S)
$$

It follows from (3.1) that the non-negative self-adjoint operator

$$
A f=A\left(\sum_{n=0}^{\infty} c_{n} \mathrm{e}_{n}\right)=\sum_{n=1}^{\infty} n^{2 \beta} c_{n} \mathrm{e}_{n}
$$

with the domain $\mathcal{D}(A)=\left\{f=\sum_{n=0}^{\infty} c_{n} \mathrm{e}_{n}:\left\{c_{n}\right\}_{n=1}^{\infty},\left\{n^{2 \beta} c_{n}\right\}_{n=1}^{\infty} \in \ell_{2}(\mathbb{N})\right\}$ is an extension of $S$.

\footnotetext{
${ }^{3}$ The concept of $\mathcal{C}$-symmetry is widely used in $\mathcal{P} \mathcal{T}$-symmetric quantum mechanics $[7,10]$.
} 
If $\beta \leq 0$, then the semi-regular sequence $\left\{\phi_{n}\right\}$ cannot be a GRS. Indeed, in this case, the operator $A$ is bounded. Therefore, $A$ coincides with the closure $\bar{S}$ of $S$. In view of (3.2), $\bar{S} \mathrm{e}_{0}=A \mathrm{e}_{0}=0$. By Theorem 2.5, $\left\{\phi_{n}\right\}$ cannot be a GRS.

Assume now that $\beta>0$. Then $A$ is an unbounded non-negative self-adjoint extension of $S$. Hence, $A$ is an extension of $\bar{S}$. Using (3.1) and (3.2), we obtain that $f=\sum_{n=0}^{\infty} c_{n} \mathrm{e}_{n}$ belongs to $\mathcal{D}(\bar{S})$ if and only if

$$
\left\{c_{n}\right\},\left\{n^{2 \beta} c_{n}\right\} \in \ell_{2}(\mathbb{N}), \text { the series } \sum_{n=1}^{\infty} \frac{n^{\beta} c_{n}}{n^{\alpha}} \text { converges and } c_{0}=\sum_{n=1}^{\infty} \frac{n^{\beta} c_{n}}{n^{\alpha}} .
$$

Since $(\bar{S} f, f)=\sum_{n=1}^{\infty} n^{2 \beta}\left|c_{n}\right|^{2}$, the operator $\bar{S}$ is positive. Using statement (iii) of Theorem 2.5 we show that the semi-regular sequence $\left\{\phi_{n}\right\}$ is a GRS for $\alpha>\frac{1}{2}$. To that end, it suffices to verify the implication (2.5).

Let $f_{m}=\sum_{n=0}^{\infty} c_{n}^{m} \mathrm{e}_{n}$ be a sequence of elements $f_{m} \in \mathcal{D}(\bar{S})$ satisfying (2.5). Then

$$
\lim _{m \rightarrow \infty}\left(\bar{S} f_{m}, f_{m}\right)=\lim _{m \rightarrow \infty} \sum_{n=1}^{\infty} n^{2 \beta}\left|c_{n}^{m}\right|^{2}=\left\|\left\{n^{\beta} c_{n}^{m}\right\}\right\|_{\ell_{2}(\mathbb{N})}^{2}=0
$$

and, since $\left\{1 / n^{\alpha}\right\} \in \ell_{2}(\mathbb{N})$ for $\alpha>\frac{1}{2}$,

$$
\begin{aligned}
g & =\lim _{m \rightarrow \infty} f_{m}=\lim _{m \rightarrow \infty} \sum_{n=1}^{\infty} c_{n}^{m} \mathrm{e}_{n}+\lim _{m \rightarrow \infty} \sum_{n=1}^{\infty} \frac{n^{\beta} c_{n}^{m}}{n^{\alpha}} \mathrm{e}_{0} \\
& =\lim _{m \rightarrow \infty}\left(\left\{n^{\beta} c_{n}^{m}\right\},\left\{1 / n^{\alpha}\right\}\right)_{\ell_{2}(\mathbb{N})} \mathrm{e}_{0}=0
\end{aligned}
$$

that justifies the implication (2.5).

\subsection{A Semi-regular Bessel Sequence}

Let $\left\{\mathrm{e}_{n}\right\}_{\mathbb{Z}}$ be an ONB of $\mathcal{H}$. Denote $\phi_{n}=K \mathrm{e}_{n}, n \in \mathbb{Z} \backslash\{0\}$, where $K$ is a bounded operator in $\mathcal{H}$. The sequence $\left\{\phi_{n}\right\}_{\mathbb{Z} \backslash\{0\}}$ is a Bessel sequence since

$$
\sum_{n \neq 0}\left|\left(f, \phi_{n}\right)\right|^{2}=\sum_{n \neq 0}\left|\left(K^{*} f, \mathrm{e}_{n}\right)\right|^{2} \leq\left\|K^{*} f\right\|^{2} \leq\left\|K^{*}\right\|^{2}\|f\|^{2}
$$

\section{Proposition 3.1 The following are equivalent:}

(i) $\left\{\phi_{n}\right\}$ is semi-regular in $\mathcal{H}$;

(ii) $\operatorname{ker} K^{*}=\{0\}$ and there exists a unique sequence of numbers $\left\{\alpha_{n}\right\}_{\mathbb{Z} \backslash\{0\}}$ such that $e_{n}-\alpha_{n} e_{0} \in \mathcal{R}\left(K^{*}\right)$.

Proof (i) $\rightarrow$ (ii) In view of the equality $\left(h, \phi_{n}\right)=\left(K^{*} h, \mathrm{e}_{n}\right)$ where $n \in \mathbb{Z} \backslash\{0\}$, the completeness of $\left\{\phi_{n}\right\}$ is equivalent to the conditions: $\operatorname{ker} K^{*}=\{0\}$ and $\mathrm{e}_{0} \notin \mathcal{R}\left(K^{*}\right)$. Let $\left\{\psi_{n}\right\}$ be the bi-orthogonal sequence for $\left\{\phi_{n}\right\}$. Then 


$$
\delta_{n m}=\left(\phi_{n}, \psi_{m}\right)=\left(\mathrm{e}_{n}, K^{*} \psi_{m}\right)
$$

that means $K^{*} \psi_{m}=\mathrm{e}_{m}+\alpha_{m} \mathrm{e}_{0}$, where $\alpha_{m}$ is determined uniquely (since $\mathrm{e}_{0} \notin$ $\left.\mathcal{R}\left(K^{*}\right)\right)$.

(ii) $\rightarrow$ (i) The vector $\mathrm{e}_{0}$ does not belong to $\mathcal{R}\left(K^{*}\right)$ since the sequence $\left\{\alpha_{n}\right\}$ is determined uniquely in the relation $\mathrm{e}_{n}-\alpha_{n} \mathrm{e}_{0} \in \mathcal{R}\left(K^{*}\right)$. This fact and $\operatorname{ker} K^{*}=\{0\}$ give the completeness of $\left\{\phi_{n}\right\}$ in $\mathcal{H}$. The minimality of $\left\{\phi_{n}\right\}$ follows from the fact that $\left\{\psi_{n}=K^{*-1}\left(\mathrm{e}_{n}-\alpha_{n} \mathrm{e}_{0}\right)\right\}$ is a bi-orthogonal sequence for $\left\{\phi_{n}\right\}$.

In view of Theorem 2.13, the sequence $\left\{\phi_{n}\right\}$ is a GRS if and only if the condition (ii) of Proposition 3.1 holds. In this case, $\left\{\phi_{n}=e^{Q / 2} e_{n}\right\}_{\mathbb{Z} \backslash\{0\}}$, where $Q$ is a self-adjoint operator such that $\sigma(Q) \subset(-\infty, 2 \ln \|K\|]$ and $\left\{e_{n}\right\}_{\mathbb{Z} \backslash\{0\}}$ is an ONB of $\mathcal{H}$.

In the space $\mathcal{H}=L_{2}(0,1)$, the operator $K f=x^{N} f(x)(N \in \mathbb{N})$ is bounded. Consider the sequence $\left\{\phi_{n}=x^{N} \mathrm{e}_{n}\right\}_{\mathbb{Z} \backslash\{0\}}$, where $\left\{\mathrm{e}_{n}=e^{2 \pi i n x}\right\}_{\mathbb{Z}}$ is the trigonometric ONB of $\mathcal{H}=L_{2}(0,1)$. It is easy to see that the condition (ii) of Proposition 3.1 holds for $N=1$ only (then $\alpha_{n}=1$ for all $n \in \mathbb{Z} \backslash\{0\}$ ). Therefore, the sequence $\left\{\phi_{n}=x^{N} e^{2 \pi i n x}\right\}_{\mathbb{Z} \backslash\{0\}}$ is a GRS for $N=1$, see [15, p.158] and [28].

\subsection{J-Orthonormal Sequences of the First/Second type}

Let a sequence of real numbers $\left\{\alpha_{k}\right\}_{k=0}^{\infty}$ satisfy the conditions

$$
0 \leq \alpha_{0}<\alpha_{1}<\alpha_{2} \ldots, \quad \lim _{k \rightarrow \infty} \alpha_{k}=\infty
$$

and let $\left\{\mathrm{e}_{n}\right\}_{n=0}^{\infty}$ be an ONB of $\mathcal{H}$ such that $J \mathrm{e}_{n}=(-1)^{n} \mathrm{e}_{n}$.

Each pair of orthonormal vectors $\left\{\mathrm{e}_{2 k}, \mathrm{e}_{2 k+1}\right\}_{k=0}^{\infty}$ can be identified with $\mathbb{C}^{2}$ assuming that

$$
U \mathrm{e}_{2 k}=\left[\begin{array}{c}
1 \\
0
\end{array}\right], \quad U \mathrm{e}_{2 k+1}=\left[\begin{array}{l}
0 \\
1
\end{array}\right]
$$

The operator $U$ is an isometric mapping of the space $\mathcal{H}_{k}=\operatorname{span}\left\{\mathrm{e}_{2 k}, \mathrm{e}_{2 k+1}\right\}$ onto $\mathbb{C}^{2}$ and $U J=\sigma_{3} U$, where $\sigma_{3}=\left[\begin{array}{cc}1 & 0 \\ 0 & -1\end{array}\right]$. Since $\mathcal{H}=\sum_{k=0}^{\infty} \oplus \mathcal{H}_{k}$, the operator $U$ can be extended to the isometric mapping of $\mathcal{H}$ onto the Hilbert space $\mathbb{H}$ of infinitely many copies of $\mathbb{C}^{2}: \mathbb{H}=\sum_{k=0}^{\infty} \oplus \mathbb{C}^{2}$. In the space $\mathbb{H}$, we define self-adjoint operators

$$
\begin{aligned}
\mathbb{Q} & =2 \sum_{k=0}^{\infty} \oplus \alpha_{k} \sigma_{1}, \quad e^{-\mathbb{Q}}=\sum_{k=0}^{\infty} \oplus e^{-2 \alpha_{k} \sigma_{1}}, \\
e^{\mathbb{Q} / 2} & =\sum_{k=0}^{\infty} \oplus e^{\alpha_{k} \sigma_{1}}, \quad \mathbb{J}=\sum_{k=0}^{\infty} \oplus \sigma_{3},
\end{aligned}
$$


where $\sigma_{1}=\left[\begin{array}{ll}0 & 1 \\ 1 & 0\end{array}\right]$. Theirs unitary equivalent copies in $\mathcal{H}$ are:

$$
Q=U^{-1} \mathbb{Q} U, \quad e^{-Q}=U^{-1} e^{-\mathbb{Q}} U, \quad e^{Q / 2}=U^{-1} e^{\mathbb{Q} / 2} U, \quad J=U^{-1} \mathbb{J} U .
$$

By the construction, $Q$ anticommutes with $J: J Q=-Q J$.

Consider vectors $\left\{\phi_{n}\right\}_{n=0}^{\infty}$ defined by the formulas:

$$
\begin{aligned}
\phi_{2 k} & =\cosh \alpha_{k} \mathrm{e}_{2 k}+\sinh \alpha_{k} \mathrm{e}_{2 k+1}, \quad k=0,1, \ldots \\
\phi_{2 k+1} & =\frac{c_{k}}{\sqrt{\mu_{2 k+1}}} \sum_{n=0}^{\infty} \frac{\chi_{n} \cosh \alpha_{n}}{1-\mu_{2 k+1} \cosh ^{2} \alpha_{n}}\left(\cosh \alpha_{n} \mathrm{e}_{2 n+1}+\sinh \alpha_{n} \mathrm{e}_{2 n}\right),
\end{aligned}
$$

where $\left\{\chi_{n}\right\}_{n=0}^{\infty}$ is a vector from $\ell_{2}(\mathbb{N})$ such that $\chi_{n} \neq 0$; the set of numbers $0<\mu_{1}<$ $\mu_{3}<\mu_{5} \ldots<1$ are roots of the equation

$$
\sum_{n=0}^{\infty} \frac{\left|\chi_{n} \cosh \alpha_{n}\right|^{2}}{1-\mu \cosh ^{2} \alpha_{n}}=0
$$

and

$$
c_{k}=\left(\sum_{n=0}^{\infty} \frac{\left|\chi_{n}\right|^{2} \cosh ^{4} \alpha_{n}}{\left(1-\mu_{2 k+1} \cosh ^{2} \alpha_{n}\right)^{2}}\right)^{-\frac{1}{2}}, \quad k=0,1 \ldots
$$

Theorem 3.2 Let the sequences $\left\{\alpha_{n}\right\}$ and $\left\{\chi_{n}\right\}$ satisfy the conditions above and let the sequence $\left\{\chi_{n} \cosh ^{2} \alpha_{n}\right\}$ do not belong to $\ell_{2}(\mathbb{N})$. Then the vectors $\phi_{n}$ determined by (3.8) form a complete $J$-orthonormal sequence $\left\{\phi_{n}\right\}_{n=0}^{\infty}$ of the first type if $\left\{\chi_{n} \cosh \alpha_{n}\right\} \notin$ $\ell_{2}(\mathbb{N})$ and of the second type if $\left\{\chi_{n} \cosh \alpha_{n}\right\} \in \ell_{2}(\mathbb{N})$.

For the first type sequence $\left\{\phi_{n}\right\}$ the formula $\phi_{n}=e^{Q / 2} e_{n}$ holds where $Q$ and $e^{Q / 2}$ are determined by (3.7) and an $O N B\left\{e_{n}\right\}$ has the form

$$
e_{2 k}=e_{2 k}, \quad e_{2 k+1}=\frac{c_{k}}{\sqrt{\mu_{2 k+1}}} \sum_{n=0}^{\infty} \frac{\chi_{n} \cosh \alpha_{n}}{1-\mu_{2 k+1} \cosh ^{2} \alpha_{n}} e_{2 n+1} .
$$

For the second type sequence such a choice of $Q$ and $\left\{e_{n}\right\}$ is impossible because the orthonormal system (3.11) is not dense in $\mathcal{H}$. A suitable operator $Q$ can be chosen as $Q=-\ln A_{F}$, where $A_{F}$ is the Friedrichs extension of the symmetric operator $S$ acting as $S \phi_{n}=(-1)^{n} J \phi_{n}$ on vectors $\phi_{n}$ and extended onto $\mathcal{D}(S)=\operatorname{span}\left\{\phi_{n}\right\}$ by the linearity.

The proof of Theorem 3.2 is given in Sect. 4 . 
Let us consider a particular case assuming that

$$
\tanh \alpha_{n}=\sqrt{\frac{n}{n+1}}, \quad \chi_{n}=\frac{1}{(n+1)^{\frac{\delta+1}{2}}}, \quad n \geq 0,
$$

and $0<\delta \leq 2$ (the condition $0<\delta$ guarantees that $\left\{\chi_{n}\right\} \in \ell_{2}(\mathbb{N})$ while $\delta \leq 2$ ensures that $\left.\left\{\chi_{n} \cosh ^{2} \alpha_{n}=1 /(n+1)^{\delta / 2-1 / 2}\right\} \notin \ell_{2}(\mathbb{N})\right)$. Then the root Eq. (3.9) takes the form

$$
\sum_{n=1}^{\infty} \frac{1}{n^{\delta}} \cdot \frac{1}{1-n \mu}=0
$$

$c_{k}=\left(\sum_{n=1}^{\infty} \frac{n^{1-\delta}}{\left(1-\mu_{2 k+1} n\right)^{2}}\right)^{-\frac{1}{2}}$, and the sequence $\left\{\phi_{n}\right\}_{n=0}^{\infty}$ :

$$
\begin{aligned}
\phi_{2 k} & =\sqrt{k+1} \mathrm{e}_{2 k}+\sqrt{k} \mathrm{e}_{2 k+1}, \quad k=0,1,2 \ldots \\
\phi_{2 k+1} & =\frac{c_{k}}{\sqrt{\mu_{2 k+1}}} \sum_{n=1}^{\infty} \frac{1}{n^{\delta / 2}} \cdot \frac{1}{1-n \mu_{2 k+1}}\left(\sqrt{n} \mathrm{e}_{2 n-1}+\sqrt{n-1} \mathrm{e}_{2 n-2}\right),
\end{aligned}
$$

turns out to be the first kind if $0<\delta \leq 1$ and the second kind if $1<\delta \leq 2$.

Figure 1 contains a numerical localization of the first 5 roots of (3.12).

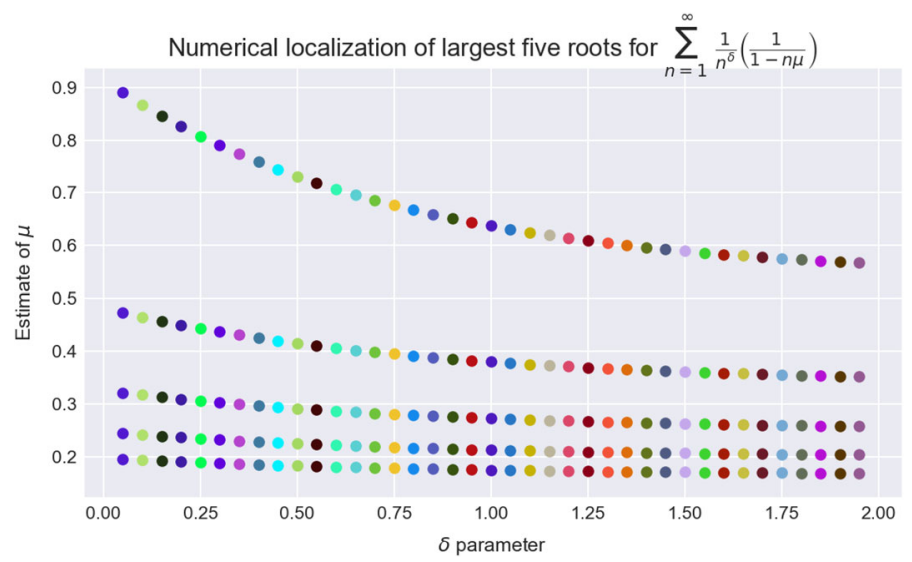

Fig. 1 First five roots to the Eq. (3.12) for different $\delta$ parameter value 
Acknowledgements This work was partially supported by the Faculty of Applied Mathematics AGH UST statutory tasks within subsidy of Ministry of Science and Higher Education.

Open Access This article is licensed under a Creative Commons Attribution 4.0 International License, which permits use, sharing, adaptation, distribution and reproduction in any medium or format, as long as you give appropriate credit to the original author(s) and the source, provide a link to the Creative Commons licence, and indicate if changes were made. The images or other third party material in this article are included in the article's Creative Commons licence, unless indicated otherwise in a credit line to the material. If material is not included in the article's Creative Commons licence and your intended use is not permitted by statutory regulation or exceeds the permitted use, you will need to obtain permission directly from the copyright holder. To view a copy of this licence, visit http://creativecommons.org/licenses/by/4.0/.

\section{Appendix: The Proof of Theorem 3.2}

We refer [8, Subsection III.1] and [20] for results of the Krein space theory which are necessary for our exposition.

\subsection{Preliminaries}

An operator $T$ defined by the formula

$$
T e_{2 k}=\tanh \alpha_{k} \mathrm{e}_{2 k+1}, \quad T e_{2 k+1}=\tanh \alpha_{k} \mathrm{e}_{2 k}, \quad k=0,1,2 \ldots
$$

on the ONB $\left\{\mathrm{e}_{n}\right\}_{n=0}^{\infty}$ and extended onto $\mathcal{H}$ by the linearity is a self-adjoint strong contraction ('strong' means that $\|T f\|<\|f\|$ for non-zero $f$ ). Moreover

$$
J T=-T J
$$

since $J e_{n}=(-1)^{n} e_{n}$. The properties of $T$ allow one to define $J$-orthogonal maximal positive $\mathfrak{L}_{+}$and maximal negative $\mathfrak{L}_{-}$subspaces of the $\operatorname{Krein}$ space $(\mathcal{H},[\cdot, \cdot])[20$, Lemma 2.2]:

$$
\mathfrak{L}_{+}=(I+T) \mathcal{H}_{+}, \quad \mathfrak{L}_{-}=(I+T) \mathcal{H}_{-},
$$

where $\mathcal{H}_{+}$and $\mathcal{H}_{-}$are the closure $($in $\mathcal{H})$ of $\operatorname{span}\left\{\mathrm{e}_{2 k}\right\}_{k=0}^{\infty}$ and $\operatorname{span}\left\{\mathrm{e}_{2 k+1}\right\}_{k=0}^{\infty}$, respectively.

Consider a vector $\chi=\sum_{k=0}^{\infty} \chi_{k} \mathrm{e}_{2 k+1}$, where $\left\{\chi_{k}\right\}$ belongs to $\ell_{2}(\mathbb{N})$. By the construction, $\chi \in \mathcal{H}_{-}$and

$$
\mathfrak{L}_{-}^{0}=\left\{(I+T) g, g \in M_{-}\right\}, \quad \text { where } M_{-}=\left\{g \in \mathcal{H}_{-},(g, \chi)=0\right\}
$$

is a subspace of the maximal negative space $\mathfrak{L}_{-}$defined by (4.3).

The $J$-orthogonal sum $\mathfrak{L}_{+} \dot{+} \mathfrak{L}_{-}$is dense in $\mathcal{H}$ since $\mathfrak{L}_{ \pm}$are maximal subspaces. However, we can not state that the set $\mathfrak{L}_{+} \dot{+} \mathfrak{L}_{-}^{0}$ remains dense in $\mathcal{H}$ since $\mathfrak{L}_{-}^{0}$ is a proper subspace of $\mathfrak{L}_{-}$.

Lemma 4.1 The sum $\mathfrak{L}_{+} \dot{+} \mathfrak{L}_{-}^{0}$ is dense in $\mathcal{H}$ if and only if $\left\{\chi_{n} \cosh ^{2} \alpha_{n}\right\} \notin \ell_{2}(\mathbb{N})$. 
Proof Assume that $h \in \mathcal{H}$ is orthogonal to $\mathfrak{L}_{+} \dot{+} \mathfrak{L}_{-}^{0}$. Then $J h$ is orthogonal to $\mathfrak{L}_{+}$with respect to the indefinite inner product $[\cdot, \cdot]$. Since $\mathfrak{L}_{-}$is the $J$-orthogonal complement of $\mathfrak{L}_{+}$, the vector $J h$ belongs to $\mathfrak{L}_{-}$. By (4.3), $J h=(I+T) f$, where $f \in \mathcal{H}_{-}$and $h=J(I+T) f=(I-T) J f=-(I-T) f$.

By the assumption, $h$ is also orthogonal to $\mathfrak{L}_{-}^{0}$. In view of (4.4) this means that $0=(h,(I+T) g)=-((I-T) f,(I+T) g)=-\left(\left(I-T^{2}\right) f, g\right)$ for all $g \in M_{-}$. Therefore, without loss of generality we can assume that $\left(I-T^{2}\right) f=\chi$. Here, $f=\sum_{k=0}^{\infty} f_{k} \mathrm{e}_{2 k+1}$ since $f \in \mathcal{H}_{-}$. In view of (4.1),

$\left(I-T^{2}\right) f=\sum_{k=0}^{\infty}\left(1-\tanh ^{2} \alpha_{k}\right) f_{k} \mathrm{e}_{2 k+1}=\sum_{k=0}^{\infty} \frac{f_{k}}{\cosh ^{2} \alpha_{k}} \mathrm{e}_{2 k+1}=\sum_{k=0}^{\infty} \chi_{k} \mathrm{e}_{2 k+1}=\chi$.

Therefore, $f_{k}=\chi_{k} \cosh ^{2} \alpha_{k}$ and we arrive at the conclusion that $\mathfrak{L}_{+} \dot{+} \mathfrak{L}_{-}^{0}$ is a nondense set in $\mathcal{H}$ if and only if $\left\{\chi_{k} \cosh ^{2} \alpha_{k}\right\}_{k=0}^{\infty} \in \ell_{2}(\mathbb{N})$.

\subsection{Complete J-Orthonormal Sequence $\left\{\phi_{n}\right\}$}

Lemma 4.2 If $\left\{\chi_{n} \cosh ^{2} \alpha_{n}\right\} \notin \ell_{2}(\mathbb{N})$, then the vectors $\left\{\phi_{n}\right\}$ defined by (3.8) form a complete $J$-orthonormal sequence in $\mathcal{H}$.

Proof The vectors $\left\{\phi_{2 k}\right\}_{k=0}^{\infty}$ in (3.8) are $J$-orthonormal because $\left[\phi_{2 k}, \phi_{2 k^{\prime}}\right]=0$ for $k \neq k^{\prime}$ and $\left[\phi_{2 k}, \phi_{2 k}\right]=\cosh ^{2} \alpha_{k}-\sinh ^{2} \alpha_{k}=1$. Moreover, in view of of (4.1), the vectors $\left\{\phi_{2 k}\right\}$ can be presented as $\phi_{2 k}=\cosh \alpha_{k}(I+T) \mathrm{e}_{2 k}$. This relation and (4.3) imply that the closure of $\operatorname{span}\left\{\phi_{2 k}\right\}_{k=0}^{\infty}$ coincides with $\mathfrak{L}_{+}$.

By virtue of (4.1),

$$
\left(I-T^{2}\right) f=\sum_{k=0}^{\infty}\left(1-\tanh ^{2} \alpha_{k}\right) f_{k} \mathrm{e}_{2 k+1} \quad \text { for all } f=\sum_{k=0}^{\infty} f_{k} \mathrm{e}_{2 k+1} \in \mathcal{H}_{-} .
$$

This relation yields that $I-T^{2}$ is a compact operator in $\mathcal{H}_{-}$, since $\lim _{k \rightarrow \infty}(1-$ $\left.\tanh ^{2} \alpha_{k}\right)=0$, see [14, problem 132]. Therefore, $P_{M_{-}}\left(I-T^{2}\right) P_{M_{-}}$, where $P_{M_{-}}$ is an orthogonal projection in $\mathcal{H}_{-}$onto the subspace $M_{-}$defined in (4.4), is a selfadjoint compact operator in $M_{-}$. This implies the existence of an ONB $\left\{\gamma_{2 k+1}\right\}$ of $M_{-}$which is formed by eigenfunctions of $P_{M_{-}}\left(I-T^{2}\right) P_{M_{-}}$. Let $\left\{\mu_{2 k+1}\right\}_{k=0}^{\infty}$ be the corresponding eigenvalues, i.e., $P_{M_{-}}\left(I-T^{2}\right) \gamma_{2 k+1}=\mu_{2 k+1} \gamma_{2 k+1}$. Since $I-T^{2}$ is a positive contraction, we can state that $0<\mu_{2 k+1}<1$ and $\lim _{k \rightarrow \infty} \mu_{2 k+1}=0$.

Denote

$$
\phi_{2 k+1}=\frac{1}{\sqrt{\mu_{2 k+1}}}(I+T) \gamma_{2 k+1}, \quad k=0,1 \ldots
$$

The vectors $\left\{\phi_{2 k+1}\right\}_{k=0}^{\infty}$ are $J$-orthonormal because

$$
\left[\phi_{2 k+1}, \phi_{2 k^{\prime}+1}\right]=-\frac{\left(\left(I-T^{2}\right) \gamma_{2 k+1}, \gamma_{2 k^{\prime}+1}\right)}{\sqrt{\mu_{2 k+1} \mu_{2 k^{\prime}+1}}}
$$




$$
=-\sqrt{\frac{\mu_{2 k+1}}{\mu_{2 k^{\prime}+1}}}\left(\gamma_{2 k+1}, \gamma_{2 k^{\prime}+1}\right)=-\delta_{k k^{\prime}}
$$

Moreover, in view of (4.4), $\phi_{2 k+1} \in \mathfrak{L}_{-}^{0}$ and the closure of $\operatorname{span}\left\{\phi_{2 k+1}\right\}_{k=0}^{\infty}$ coincides with $\mathfrak{L}_{-}^{0}$. Applying now Lemma 4.1 we arrive at the conclusion that, for the case $\left\{\chi_{n} \cosh ^{2} \alpha_{n}\right\} \notin \ell_{2}(\mathbb{N})$, the $J$-orthonormal sequence $\left\{\phi_{n}\right\}_{n=0}^{\infty}$, where $\left\{\phi_{2 k}\right\}_{k=0}^{\infty}$ and $\left\{\phi_{2 k+1}\right\}_{k=0}^{\infty}$ are defined by (3.8) and (4.5), respectively, is complete in $\mathcal{H}$.

To finish the proof of Lemma 4.2 it suffices to show that the formulas (3.8) and (4.5) determine the same vectors $\left\{\phi_{2 k+1}\right\}_{k=0}^{\infty}$. To do that, we describe the eigenvalues $\mu_{2 k+1}$ and the normalized eigenfunctions $\gamma_{2 k+1}$ of the equation

$$
P_{M_{-}}\left(I-T^{2}\right) g=\mu g, \quad g \in M_{-} .
$$

In view of (4.4), the condition $g \in M_{-}$means that

$$
\sum_{k=0}^{\infty} g_{k} \bar{\chi}_{k}=0, \text { where } g=\sum_{k=0}^{\infty} g_{k} \mathrm{e}_{2 k+1}
$$

Let $f=\sum_{k=0}^{\infty} f_{k} \mathrm{e}_{2 k+1}$ be an arbitrary element of $\mathcal{H}_{-}$. Then

$$
P_{M_{-}} f=\sum_{k=0}^{\infty}\left(f_{k}-\alpha[f] \chi_{k}\right) \mathrm{e}_{2 k+1}, \quad \alpha[f]=\frac{1}{\|\chi\|^{2}} \sum_{k=0}^{\infty} f_{k} \bar{\chi}_{k} .
$$

Using (4.9) we rewrite (4.7) as

$$
\begin{aligned}
P_{M_{-}} & \sum_{k=0}^{\infty}\left(1-\tanh ^{2} \alpha_{k}\right) g_{k} \mathrm{e}_{2 k+1} \\
& =\sum_{k=0}^{\infty}\left[\left(1-\tanh ^{2} \alpha_{k}\right) g_{k}-\alpha\left[\left(I-T^{2}\right) g\right] \chi_{k}\right] \mathrm{e}_{2 k+1}=\mu \sum_{k=0}^{\infty} g_{k} \mathrm{e}_{2 k+1}
\end{aligned}
$$

that implies

$$
\left(1-\tanh ^{2} \alpha_{k}-\mu\right) g_{k}=\alpha\left[\left(I-T^{2}\right) g\right] \chi_{k}, \quad k=0,1, \ldots
$$

It is important that $\alpha\left[\left(I-T^{2}\right) g\right] \neq 0$ in (4.10). Indeed, if $\alpha\left[\left(I-T^{2}\right) g\right]=0$, then $\left(1-\tanh ^{2} \alpha_{k}-\mu\right) g_{k}=0$ for all $k$. Due to conditions imposed on $\alpha_{n}$ in (3.4), there exists a unique $k^{\prime}$ such that $g_{k^{\prime}} \neq 0$ and $\mu=1-\tanh ^{2} \alpha_{k^{\prime}}$. This means that $g=g_{k^{\prime}} \mathrm{e}_{2 k^{\prime}+1}$ belongs to $M_{-}$. The last fact is impossible because $0=<g, \chi>=g_{k^{\prime}} \bar{\chi}_{k^{\prime}} \neq 0$ (we recall that $\chi_{n} \neq 0$ for all $n$ by the assumption). The obtained contradiction shows that $\alpha\left[\left(I-T^{2}\right) g\right] \neq 0$. This means that $\left(1-\tanh ^{2} \alpha_{k}-\mu\right) g_{k} \neq 0$ and (4.10) can be rewritten as

$$
g_{k}=\alpha\left[\left(I-T^{2}\right) g\right] \frac{\chi_{k} \cosh ^{2} \alpha_{k}}{1-\mu \cosh ^{2} \alpha_{k}}, \quad k=0,1,2 \ldots
$$


The corresponding solution $g(\mu)=\sum_{k=0}^{\infty} g_{k} \mathrm{e}_{2 k+1}$ of (4.7) must be in $M_{-}$. By virtue of (4.8), $g(\mu)$ belongs to $M_{-}$if and only if $\mu$ is the root of (3.9). The Eq. (3.9) has infinitely many roots $0<\mu_{1}<\mu_{3}<\ldots<\mu_{2 k+1} \ldots<1$ that coincide with eigenvalues of $P_{M_{-}}\left(I-T^{2}\right) P_{M_{-}}$. The eigenfunctions of $P_{M_{-}}\left(I-T^{2}\right) P_{M_{-}}$ corresponding to $\mu_{2 k+1}$ have the form

$$
g\left(\mu_{2 k+1}\right)=\alpha\left[\left(I-T^{2}\right) g\left(\mu_{2 k+1}\right)\right] \sum_{n=0}^{\infty} \frac{\chi_{n} \cosh ^{2} \alpha_{n}}{1-\mu_{2 k+1} \cosh ^{2} \alpha_{n}} \mathrm{e}_{2 n+1}, \quad k=0,1 \ldots
$$

Then

$$
\gamma_{2 k+1}=\frac{g\left(\mu_{2 k+1}\right)}{\left\|g\left(\mu_{2 k+1}\right)\right\|}=c_{k} \sum_{n=0}^{\infty} \frac{\chi_{n} \cosh ^{2} \alpha_{n}}{1-\mu_{2 k+1} \cosh ^{2} \alpha_{n}} \mathrm{e}_{2 n+1}, \quad k=0,1 \ldots
$$

where the normalizing factor $c_{k}$ is defined in (3.10). By the construction, $\left\{\gamma_{2 k+1}\right\}_{k=0}^{\infty}$ is an ONB of $M_{-}$. Substituting the obtained expression for $\gamma_{2 k+1}$ into (4.5) and taking (4.1) into account, we obtain the vectors $\left\{\phi_{2 k+1}\right\}$ from (3.8).

Lemma 4.3 If $\left\{\chi_{n} \cosh \alpha_{n}\right\} \notin \ell_{2}(\mathbb{N})$, then the sequence $\left\{\phi_{n}\right\}$ is of the first type. The corresponding operator $Q$ in Definition 1.1 is defined by (3.7) while $O N B\left\{e_{n}\right\}$ has the form (3.11).

Proof If $\left\{\chi_{n} \cosh \alpha_{n}\right\} \notin \ell_{2}(\mathbb{N})$, then $\left\{\chi_{n} \cosh ^{2} \alpha_{n}\right\} \notin \ell_{2}(\mathbb{N})$ and, by Lemma 4.2, $\left\{\phi_{n}\right\}$ is a complete $J$-orthonormal sequence. In view of (2.9), the operator $S$ defined by (2.4) acts as $S \phi_{n}=(-1)^{n} J \phi_{n}$. On the other hand, taking the relation $e^{-2 \alpha_{k} \sigma_{1}}=$ $\cosh 2 \alpha_{k} \sigma_{0}-\sinh 2 \alpha_{k} \sigma_{1}$ into account, we directly verify that $e^{-Q_{\phi_{n}}}=(-1)^{n} J \phi_{n}$, where $e^{-Q}$ is defined by (3.7). Therefore, $e^{-Q}$ is a positive self-adjoint extension of $S$. Denote $e_{n}=e^{-Q / 2} \phi_{n}$. In view of (3.6),

$$
\begin{aligned}
e^{-Q / 2} & =U^{-1} \sum_{k=0}^{\infty} \oplus\left[\cosh \alpha_{k} \sigma_{0}-\sinh \alpha_{k} \sigma_{1}\right] U \\
& =U^{-1} \sum_{k=0}^{\infty} \oplus\left[\begin{array}{cc}
\cosh \alpha_{k} & -\sinh \alpha_{k} \\
-\sinh \alpha_{k} & \cosh \alpha_{k}
\end{array}\right] U .
\end{aligned}
$$

This expression, (3.5), and (3.8) allow one to calculate $\left\{e_{n}\right\}$ precisely, as (3.11).

By analogy with the proof of Theorem 2.5 we obtain that $\left\{e_{n}\right\}$ is an orthonormal sequence in $\mathcal{H}$. Moreover, $\left\{e_{n}\right\}$ is an ONB if and only if $\left\{\phi_{n}\right\}$ is complete in the Hilbert space $\mathcal{H}_{-Q}$. Below we show that the completeness of $\left\{\phi_{n}\right\}$ in $\mathcal{H}_{-Q}$ is equivalent to the condition $\left\{\chi_{n} \cosh \alpha_{n}\right\} \notin \ell_{2}(\mathbb{N})$.

We begin with the remark that

$$
e^{-Q}=(I-T)(I+T)^{-1}
$$

where $T$ is determined by (4.1). 
Indeed, since the subspaces $\mathcal{H}_{k}=\operatorname{span}\left\{\mathrm{e}_{2 k}, \mathrm{e}_{2 k+1}\right\}$ are invariant with respect to $T$ and $U$ satisfies (3.5), we get that $\left.U T U^{-1}\right|_{\mathcal{H}_{k}}$ acts as the multiplication by $\tanh \alpha_{k} \sigma_{1}$ in $\mathbb{C}^{2}$ and $\left.U(I-T)(I+T)^{-1} U^{-1}\right|_{\mathcal{H}_{k}}$ coincides with

$$
\begin{aligned}
{\left[\begin{array}{cc}
\cosh \alpha_{k} & -\sinh \alpha_{k} \\
-\sinh \alpha_{k} & \cosh \alpha_{k}
\end{array}\right]^{2}=\left[\begin{array}{cc}
\cosh 2 \alpha_{k} & -\sinh 2 \alpha_{k} \\
-\sinh 2 \alpha_{k} & \cosh 2 \alpha_{k}
\end{array}\right] } \\
=\left(\cosh 2 \alpha_{k} \sigma_{0}-\sinh 2 \alpha_{k} \sigma_{1}\right)^{2}=e^{-2 \alpha_{k} \sigma_{1}}
\end{aligned}
$$

This relation and the decomposition $\mathcal{H}=\sum_{k=0}^{\infty} \oplus \mathcal{H}_{k}$ justify (4.11).

The formulas (4.3) and (4.11) lead to the conclusion that $\mathcal{D}\left(e^{-Q}\right)=\mathfrak{L}_{+} \dot{+} \mathfrak{L}_{-}$, where the subspaces $\mathfrak{L}_{ \pm}$are orthogonal with respect to the inner product (2.3). Therefore, the space $\mathcal{H}_{-Q}$ has the decomposition

$$
\mathcal{H}_{-Q}=\widehat{\mathfrak{L}}_{+} \dot{+} \widehat{\mathfrak{L}}_{-}
$$

where the subspaces $\widehat{\mathfrak{L}}_{ \pm}$are the completions of linear manifolds $\mathfrak{L}_{ \pm}$in $\mathcal{H}_{-Q}$.

To prove the completeness of $\left\{\phi_{n}\right\}$ in $\mathcal{H}_{-Q}$ we note that $\left\{\phi_{2 k}\right\}$ is a complete set in $\widehat{\mathfrak{L}}_{+}$. This fact can be justified as follows: due to the proof of Lemma 4.2, $\operatorname{span}\left\{\phi_{2 k}\right\}$ is dense in the subspace $\mathfrak{L}_{+}$of $\mathcal{H}$. In view of (4.2), (4.3), and (4.11),

$$
\|f\|_{-Q}^{2}=\left(e^{-Q} f, f\right)=\left((I-T) x_{+},(I+T) x_{+}\right)=[f, f] \leq\|f\|^{2}
$$

for each $f=(I+T) x_{+} \in \mathfrak{L}_{+}$. Therefore, each $f \in \mathfrak{L}_{+}$can be approximated by vectors from $\operatorname{span}\left\{\phi_{2 k}\right\}$ with respect to the norm $\|\cdot\|_{-Q}$. Since $\widehat{\mathfrak{L}}_{+}$is the completion of $\mathfrak{L}_{+}$in $\mathcal{H}_{-Q}$, the set $\left\{\phi_{2 k}\right\}$ is complete in $\widehat{\mathfrak{L}}_{+}$.

Similar arguments and the fact that $\operatorname{span}\left\{\phi_{2 k+1}\right\}$ is dense in the subspace $\mathfrak{L}_{-}^{0}$ lead to the conclusion that each vector $f \in \mathfrak{L}_{-}^{0}$ can be approximated by vectors of $\operatorname{span}\left\{\phi_{2 k+1}\right\}$ with respect to $\|\cdot\|_{-Q}$. Therefore, in order to proof the completeness of $\left\{\phi_{2 k+1}\right\}$ in $\widehat{\mathfrak{L}}_{-}$it suffices to find when $\mathfrak{L}_{-}^{0}$ turns out to be dense in $\widehat{\mathfrak{L}}_{-}$with respect to $\|\cdot\|_{-Q}$.

Let $h \in \widehat{\mathfrak{L}}_{-}$be orthogonal to $\mathfrak{L}_{-}^{0}$ in $\mathcal{H}_{-Q}$. Since $\mathfrak{L}_{-}$is dense in $\widehat{\mathfrak{L}}_{-}$we can approximate $h$ by a sequence $\left\{f_{n}\right\}$, where $f_{n} \in \mathfrak{L}_{-}$. In view of (2.3), the sequence $\left\{e^{-Q / 2} f_{n}\right\}$ is fundamental in $\mathcal{H}$ and, hence, $\lim _{n \rightarrow \infty} e^{-Q / 2} f_{n}=f \in \mathcal{H}$. Due to (4.3), $f_{n}=(I+T) x_{-}^{n}$, where $x_{-}^{n} \in \mathcal{H}_{-}$. Moreover, $e^{-Q / 2}=\left[(I-T)(I+T)^{-1}\right]^{1 / 2}$ in view of (4.11). This means that

$$
e^{-Q / 2} f_{n}=\left[(I-T)(I+T)^{-1}\right]^{1 / 2}(I+T) x_{-}^{n}=\left(I-T^{2}\right)^{1 / 2} x_{-}^{n}
$$

and, since $\left(I-T^{2}\right)^{1 / 2}$ leaves $\mathcal{H}_{ \pm}$invariant, $f=\lim _{n \rightarrow \infty} e^{-Q / 2} f_{n}=\lim _{n \rightarrow \infty}(I-$ $\left.T^{2}\right)^{1 / 2} x_{-}^{n}=f \in \mathcal{H}_{-}$. On the other hand, for each vector $(I+T) g \in \mathfrak{L}_{-}^{0}$, the relation $e^{-Q / 2}(I+T) g=\left(I-T^{2}\right)^{1 / 2} g$ holds. After such kind of auxiliary work we obtain:

$$
\begin{aligned}
0 & =(h,(I+T) g)_{-Q}=\lim _{n \rightarrow \infty}\left(f_{n},(I+T) g\right)_{-Q}=\lim _{n \rightarrow \infty}\left(e^{-Q / 2} f_{n}, e^{-Q / 2}(I+T) g\right) \\
& =\left(f,\left(I-T^{2}\right)^{1 / 2} g\right)=\left(\left(I-T^{2}\right)^{1 / 2} f, g\right) \text { for all } g \in M_{-} .
\end{aligned}
$$


Therefore, without loss of generality we can assume that $\left(I-T^{2}\right)^{1 / 2} f=\chi$. Reasoning by analogy with the final part of the proof of Lemma 4.1 , we obtain that $\mathfrak{L}_{-}^{0}$ is dense in $\widehat{\mathfrak{L}}_{-}$if and only if $\left\{\chi_{n} \cosh \alpha_{n}\right\} \notin \ell_{2}(\mathbb{N})$. This relation guarantees the completeness of $\left\{\phi_{n}\right\}$ in $\mathcal{H}_{-Q}$.

\subsection{The Proof of Theorem 3.2}

The implication ' $\left\{\chi_{n} \cosh \alpha_{n}\right\} \notin \ell_{2}(\mathbb{N}) \rightarrow\left\{\phi_{n}\right\}$ is a first type sequence' was proved in Lemma 4.3. Let us assume that $\left\{\phi_{n}\right\}$ is of the first type, i.e., there exists a self-adjoint operator $Q^{\prime}$ anti-commuting with $J$ and such that $\phi_{n}=e^{Q^{\prime} / 2} e_{n}^{\prime}$, where $\left\{e_{n}^{\prime}\right\}$ is an ONB of $\mathcal{H}$.

Denote $\mathcal{C}=J e^{-Q^{\prime}}$. Since $Q^{\prime}$ anti-commutes with $J$, the operator $\mathcal{C}$ satisfies the relation $\mathcal{C}^{2} f=f$ for $f \in \mathcal{D}(\mathcal{C})$ and $J \mathcal{C}=e^{-Q^{\prime}}$ is a positive self-adjoint operator in $\mathcal{H}$. In view of [7, Theorem 6.2.3], there exists $J$-orthonormal maximal positive $\mathfrak{L}_{+}^{\prime}$ and maximal negative $\mathfrak{L}_{-}^{\prime}$ subspaces of the Krein space $(\mathcal{H},[\cdot, \cdot])$ which uniquely characterize $\mathcal{C}$ in the following way: $\mathcal{C} f_{+}=f_{+}$and $\mathcal{C} f_{-}=f_{-}$for $f_{ \pm} \in \mathfrak{L}_{ \pm}^{\prime}$.

Since $e^{-Q^{\prime}}$ is an extension of $S$, we obtain $\mathcal{C} \phi_{n}=J e^{-Q^{\prime}} \phi_{n}=J S \phi_{n}=(-1)^{n} \phi_{n}$. Therefore, the operator $\mathcal{C}$ acts as the identity operator on elements of the subspace $\mathfrak{L}_{+}$defined by (4.3) (since $\operatorname{span}\left\{\phi_{2 k}\right\}_{k=0}^{\infty}$ is dense in $\left.\mathfrak{L}_{+}\right)$. This yields that $\mathfrak{L}_{+}^{\prime}=\mathfrak{L}_{+}$ and, moreover $\mathfrak{L}_{-}^{\prime}=\mathfrak{L}_{-}$since the maximal negative subspace $\mathfrak{L}_{-}^{\prime}$ is determined uniquelly as $J$-orthogonal complement of $\mathfrak{L}_{+}^{\prime}=\mathfrak{L}_{+}$. We obtain that the $J$-orthogonal sum $\mathfrak{L}_{+} \dot{+} \mathfrak{L}_{-}$determines two operators $J e^{-Q^{\prime}}$ and $J e^{-Q}$. Applying again [7, Theorem 6.2.3], we conclude that $Q^{\prime}=Q$, where $Q$ is determined by (3.7). In this case, $e_{n}^{\prime}=e^{-Q^{\prime} / 2} \phi_{n}=e^{-Q / 2} \phi_{n}=e_{n}$, where $\left\{e_{n}\right\}$ is determined by (3.11). Therefore, $\left\{e_{n}\right\}$ is an ONB of $\mathcal{H}$ that, in view of the proof of Lemma 4.3 is equivalent to the condition $\left\{\chi_{n} \cosh \alpha_{n}\right\} \notin \ell_{2}(\mathbb{N})$. The inverse implication 'first type sequence $\left\{\phi_{n}\right\} \rightarrow\left\{\chi_{n} \cosh \alpha_{n}\right\} \notin \ell_{2}(\mathbb{N}) '$ is proved.

If $\left\{\phi_{n}\right\}$ is second type, the choice of $Q$ as in (3.7) leads to the non-complete orthonormal sequence (3.11) in $\mathcal{H}$. Trying to keep the GRS's formula $\phi_{n}=e^{Q / 2} e_{n}$ we have to use $Q=-\ln A$, where $A$ is a positive extremal extension of $S$ (without loss of generality, we may assume that $A=A_{F}$ ). In this case, the ONB $\left\{e_{n}\right\}$ will be different from (3.11).

\section{References}

1. Ando, T., Nishio, K.: Positive self-adjoint operators of positive symmetric operators. Tohoku Math. J. 22, 65-75 (1970)

2. Arlinskiŭ, Y.M., Hassi, S., Sebestyén, Z., de Snoo, H.S.V.: On the class of extremal extensions of a nonnegative operator. In: Kerchy, L., Foias, C.I., Gohberg, I., Langer, H. (eds.) Recent Advances in Operator Theory and Related Topics the Bela Szokefalvi-Nagy Memorial Volume, Operator Theory: Advances and Applications, vol. 127, pp. 41-81. Basel, Birkhäuser (2001)

3. Arlinskiı̆, Y.M., Tsekanovskiǔ, E.: M. Krein's research on semi-bounded operators, its contemporary developments, and applications. In: Adamyan, V.M., et al. (eds.) Modern Analysis and Applications. Operator Theory: Advances and Applications, vol. 190, pp. 65-112. Basel, Birkhäuser (2009)

4. Bagarello, F.: More mathematics on pseudo-bosons. J. Math. Phys. 54, 063512 (2013)

5. Bagarello, F., Bellomonte, G.: Hamiltonians defined by biorthogonal sets. J. Phys. A 50, 145203 (2017) 
6. Bagarello, F., Inoue, H., Trapani, C.: Biorthogonal vectors, sesquilinear forms and some physical operators. J. Math. Phys. 59, 033506 (2018)

7. Bagarello, F., Gazeau, J.-P., Szafraniec, F.H., Znojil, M. (eds.): Non-selfadjoint Operators in Quantum Physics. Mathematical Aspects. Wiley, Hoboken (2015)

8. Bagarello, F., Kużel, S.: Generalized Riesz systems and orthonormal sequences in Krein spaces. J. Phys. A (2020). https://doi.org/10.1088/1751-8121/ab67e4

9. Bellomonte, G., Trapani, C.: Riesz-like bases in rigged Hilbert spaces. Z. Anal. Anwend. 35, 243-265 (2016)

10. Bender, C.M., et al.: $\mathcal{P} \mathcal{T}$-Symmetry in Quantum and Classical Physics. World Scientific, Singapore (2019)

11. Bender, C.M., Fring, A., Günther, U., Jones, H.: Special issue on quantum physics with non-Hermitian operators. J. Phys. A 45(44), 440301 (2012)

12. Christensen, O.: An Introduction to Frames and Riesz Bases. Birkhäuser, Boston (2003)

13. Davies, E.B.: Wild spectral behaviour of anharmonic oscillators. Bull. Lond. Math. Soc. 32, 432-438 (1999)

14. Halmos, P.R.: A Hilbert Space Problem Book. Springer, New York (1982)

15. Heil, C.: A Basis theory primer. In: Benedetto, J.J. (ed.) Applied and Numerical Harmonic Analysis. Birkhäuser, Boston (2011)

16. Inoue, H.: General theory of regular biorthogonal pairs and its physical operators. J. Math. Phys. 57, 083511 (2016)

17. Inoue, H.: Semi-regular biorthogonal pairs and generalized Riesz bases. J. Math. Phys. 57, 113502 (2016)

18. Inoue, H., Takakura, M.: Regular biorthogonal pairs and pseudo-bosonic operators. J. Math. Phys. 57, 083503 (2016)

19. Inoue, H., Takakura, M.: Non-self-adjoint hamiltonians defined by generalized Riesz bases. J. Math. Phys. 57, 083505 (2016)

20. Kamuda, A., Kuzhel, S., Sudilovskaja, V.: On dual definite subspaces in Krein space. Complex Anal. Oper. Theory 13, 1011-1032 (2019)

21. Kato, T.: Perturbation Theory for Linear Operators. Springer, Berlin (1966)

22. Krejčiřík, D., Siegl, P., Tater, M., Viola, J.: Pseudospectra in non-Hermitian quantum mechanics. J. Math. Phys. 56, 103513 (2015)

23. Mityagin, B., Siegl, P., Viola, J.: Differential operators admitting various rates of spectral projection growth. J. Funct. Anal. 272, 3129-3175 (2017)

24. Mostafazadeh, A.: Pseudo-Hermitian representation of quantum mechanics. Int. J. Geom. Methods Mod. Phys. 7, 1191-1306 (2010)

25. Olevskii, A.M.: On operators generating conditional bases in a Hilbert space. Math. Notes 12, 476-482 (1972)

26. Schmüdgen, K.: Unbounded Self-adjoint Operators on Hilbert Space. Springer, Dordrecht (2012)

27. Siegl, P., Krejčiřík, D.: On the metric operator for the imaginary cubic oscillator. Phys. Rev. D 86, 121702 (2012)

28. Yoon, G.J., Heil, C.: Duals of weighted exponential systems. Acta Appl. Math. 119, 97-112 (2012)

Publisher's Note Springer Nature remains neutral with regard to jurisdictional claims in published maps and institutional affiliations. 